\title{
Symptomatic Perineal Hernias: A Rare but Challenging Problem after Laparoscopic Abdominoperineal Resection for Rectal Cancers. Combined Abdominoperineal Approach and Biological Mesh Repair
}

\author{
Bodvar Ýmisson ${ }^{1}$, Ahmed Hamad ${ }^{2}$, Narendranath Govindarajah ${ }^{3}$ and Arif Khan ${ }^{2}$ \\ ${ }^{1}$ Department of General Surgery, University of Manchester, United Kingdom \\ ${ }^{2}$ Department of General Surgery, Mid Cheshire Hospitals NHS Trust, United Kingdom \\ ${ }^{3}$ Department of General Surgery, Countess of Chester Hospital, United Kingdom
}

*Corresponding author: Ahmed Hamad, Department of General Surgery, Mid Cheshire Hospitals NHS Trust, Crewe, United Kingdom, E-mail: aahhamad@gmail.com

\begin{abstract}
Perineal hernia is a rare condition that can occur postoperatively following abdominoperineal resection of the rectum or other major pelvic resections. The current literature has limited described surgical techniques for their repair given its low prevalence as a surgical complication.

In this case; we describe a 69-year-old gentleman who presented with a symptomatic perineal hernia 4 month post abdominoperineal resection for low rectal adenocarcinoma. At the time of laparotomy, the patient was found to have herniated loops of small bowel within the perineum with associated adhesions.

We propose a combined method of abdominoperineal surgical approach to repair these hernias with concurrent use of a biological mesh as a safe technique for this rare postoperative complication.
\end{abstract}

\section{Introduction}

Postoperative perineal hernia is a rare complication after abdominoperineal resection (APR), proctectomy, or pelvic exenteration. It is the protrusion of intra-abdominal contents through a defect in the pelvic floor. It may contain small bowel, large bowel, bladder, uterus and omentum [1].

Prevalence after open abdominoperineal resections is $0.34 \%$. The incidence after a laparoscopic APR remains unknown at present [2].
Various approaches have been utilised for the repair including abdominal, perineal or combined, and abdominal procedures can be performed either open or laparoscopically. Repair techniques include primary suture repair, mesh repair and repair with autogenous tissue [3].

We present a case of symptomatic perineal hernia following laparoscopic assisted APR for rectal cancer that was successfully repaired using combined abdominoperineal approach and repair using a biological mesh.

\section{Case Report}

The patient in this report is a 67-year-old male who was diagnosed with moderately differentiated adenocarcinoma of the rectum (T3b NO MO) in 2014, the lesion was $2.5 \mathrm{~cm}$ from the anal verge. His previous medical history was only remarkable for well-controlled hypertension with a performance status of 1 . After long-course neoadjuvant radiotherapy, he had undergone laparoscopic-assisted APR with formation of a permanent end colostomy. Eleven months later, on attending routine outpatient follow-up, the patient reported a protruding lump in his perineum that he had noticed over the preceding seven months. He described occasional pain and general discomfort when sitting in conjunction with aching upon standing. External examination revealed a $10 \times 8 \mathrm{~cm}$ perineal hernia covered by

Citation: Ýmisson B, Hamad A, Govindarajah N, Khan A (2018) Symptomatic Perineal Hernias: A Rare but Challenging Problem after Laparoscopic Abdominoperineal Resection for Rectal Cancers. Combined Abdominoperineal Approach and Biological Mesh Repair. Int J Surg Res Pract 5:077. doi. org/10.23937/2378-3397/1410077

Accepted: June 04, 2018; Published: June 06, 2018

Copyright: (c) 2018 Ýmisson B, et al. This is an open-access article distributed under the terms of the Creative Commons Attribution License, which permits unrestricted use, distribution, and reproduction in any medium, provided the original author and source are credited. 


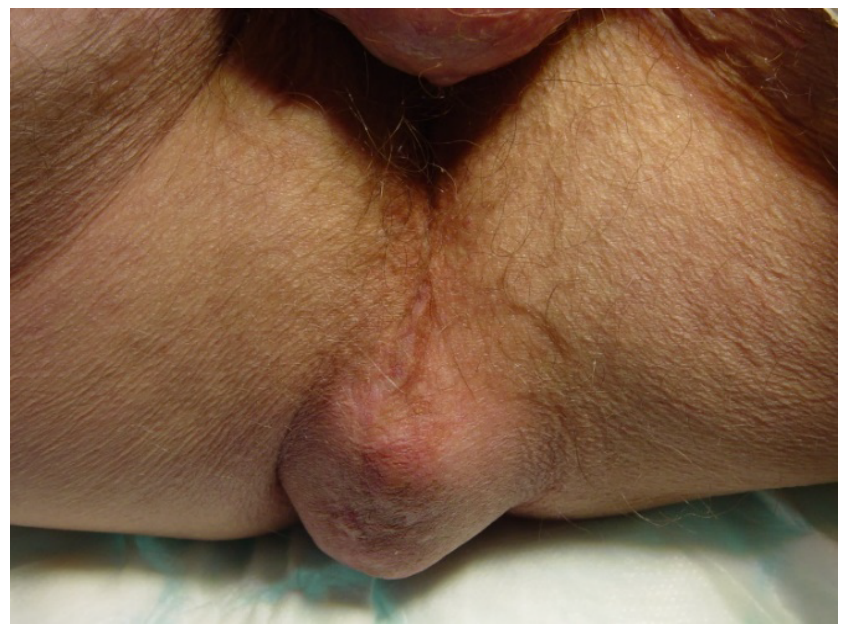

Figure 1: Clinical examination of the perineum showing hernia.

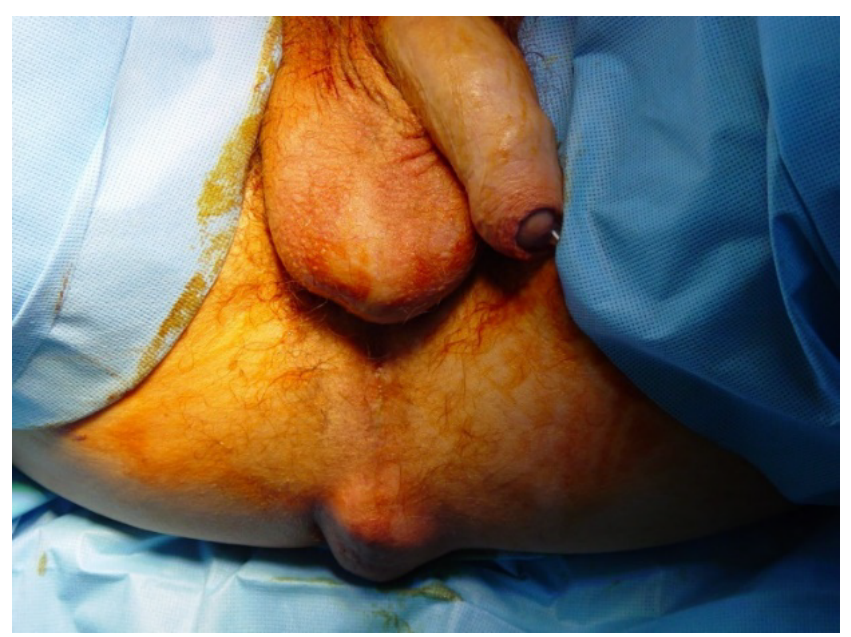

Figure 2: Perineal area prepared.

a thin, tense layer of skin which was reducible with the patient lying in the supine position (Figure 1). The cough impulse was preserved and there were no features of bowel obstruction or strangulation. The decision was made to operate electively, owing to the potential threat of skin compromise leading to development of an enterocutaneous fistula.

At operation, the patient was placed in the Lloyd Davies position (Figure 2) and a lower midline laparotomy was used to open the abdomen. Exploring the abdominal cavity revealed minimal intraperitoneal adhesions from previous surgery. In the pelvis, the small bowel had herniated through into the perineal space and formed dense adhesions which could not be visualised clearly from the abdomen. Due to high risk of bowel injury, transabdominal dissection was halted, and a midline perineal incision was made through the thin skin overlying the hernia (Figure 3). The hernial sac was opened. This revealed dense adhesions with the prostate anteriorly and right lateral pelvic wall. The small bowel was then carefully dissected from the right pelvic wall and mobilised back into the abdomen with no subsequent iatrogenic injury noted (Figure 4).

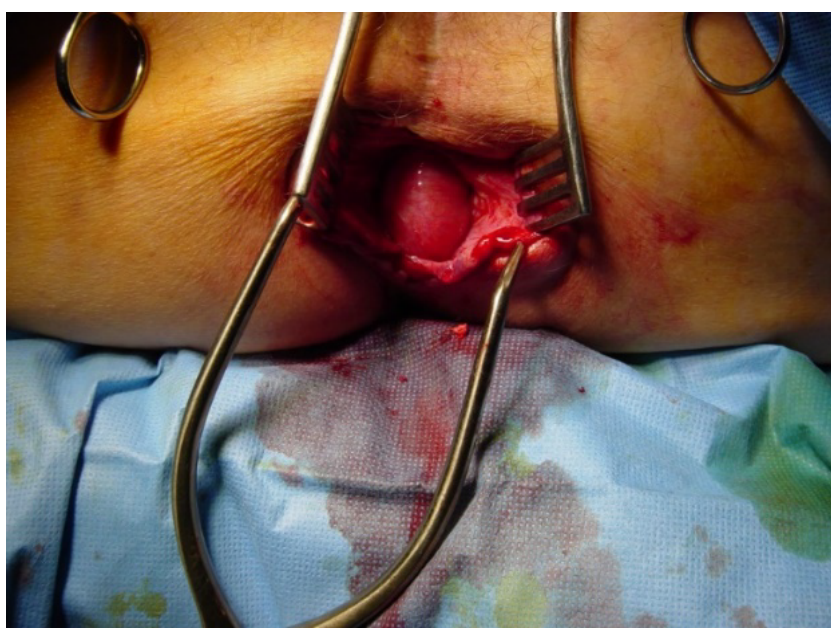

Figure 3: Hernia sac dissected from thin overlying skin.

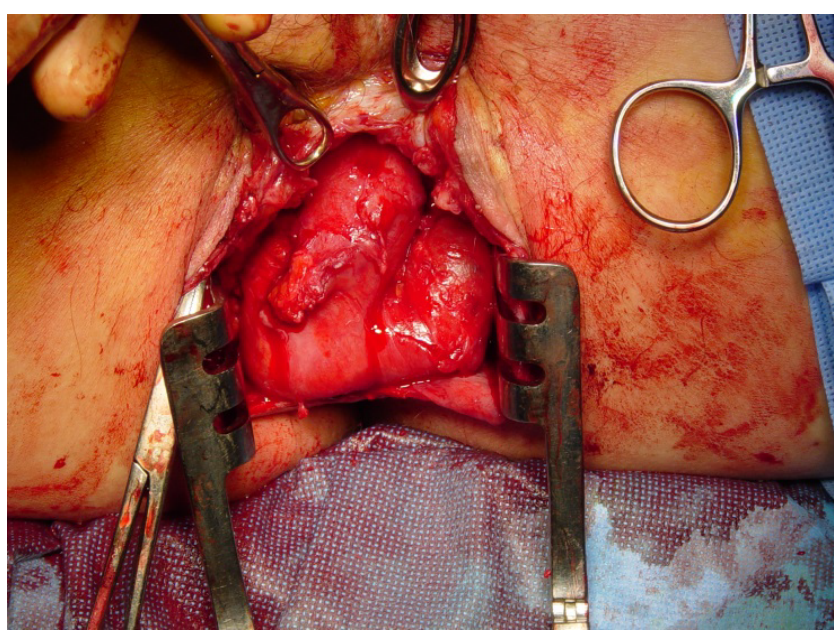

Figure 4: Small bowel freed of dense adhesions with prostate anteriorly.

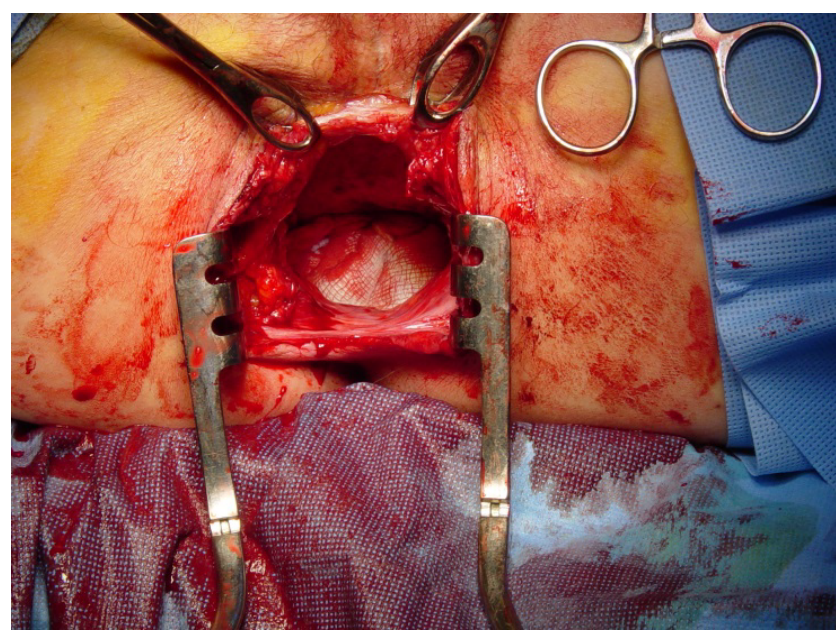

Figure 5: Small bowl reduced into pelvis. Mesh placed.

The defect was then repaired utilising a Cook Biode$\operatorname{sign}^{\text {Tm }}(10 \times 10 \mathrm{~cm})$ biological mesh (Figure 5). The mesh was sutured to the sacrum posteriorly, lateral pelvic wall fascia and anteriorly to peritoneum over the bladder (Figure 6). Normal anatomy was restored, followed by closure of the laparotomy wound and closure of the perineal wound with a perineal suction drain in place. 


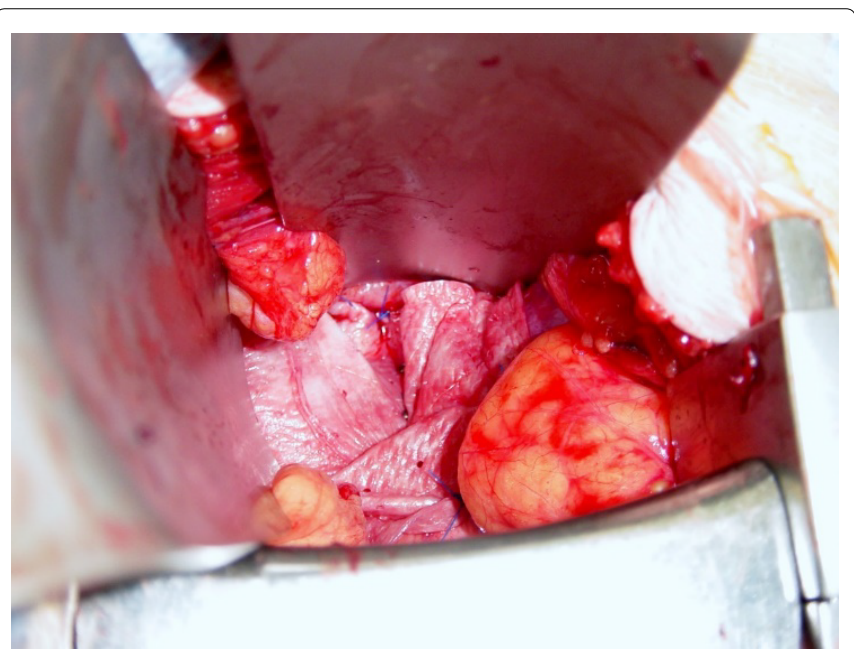

Figure 6: Mesh sutured to sacrum posteriorly, lateral pelvic wall fascia and anteriorly to peritoneum over bladder.

\section{Discussion}

Perineal hernia is an uncommon complication of abdominoperineal resection of the rectum. Prevalence estimates range from $0.34 \%$ to $7 \%$ [4].

Prevalence is thought to be higher after laparoscopic approach as this is associated with less intra-abdominal adhesions allowing abdominal contents to fall into the perineum. This can also be due to loss of pneumoperitoneum is as soon as the rectum is removed, and this is a convenient point in the operation to construct the colostomy and close the perineum, leaving the pelvic peritoneum unsutured [5].

The risk of developing such hernias is higher in females due to wider pelvis, previous hysterectomy, radiotherapy, coccygectomy, higher length of small bowel mesentery, and perineal post-operative infection.
Surgical approach to treatment can be perineal or the abdomen, with the latter being either open or laparoscopic. Alternatively, a combined abdominoperineal approach can be used.

Different repair techniques have been described including primary suturing of the perineal defect which is usually not feasible because of the size of the defect. The defect has to be strengthened with synthetic or biological meshes or autologous tissue such as peritoneal grafts, dura and fascia lata grafts, omental carpet, uterus and even the bladder [2].

In our case, an open combined abdominoperineal approach was successfully utilised, and repair was feasible using a biological mesh. The patient is still under follow up and at the time of publication there have been no post-operative complications.

\section{References}

1. Melich G, Lim DR, Hur H, Min BS, Baik SH, et al. (2016) Prevention of perineal hernia after laparoscopic and robotic abdominoperineal resection: review with illustrative case series of internal hernia through pelvic mesh. Can J Surg 59: 54-58.

2. Penkov N, Damianov D, Asenov Y, Gerzilov P, Sedloev T (2015) Recurrent perineal hernia - case report and review of the literature. Chirurgia (Bucur) 110: 81-83.

3. Douglas SR, Longo WE, Narayan D (2013) A novel technique for perineal hernia repair. BMJ Case Rep.

4. Aboian E, Winter DC, Metcalf DR, Wolff BG (2006) Perineal hernia after proctectomy: prevalence, risks, and management. Dis Colon Rectum 49: 1564-1568.

5. Ewan LC, Charleston PJ, Pettit SH (2014) Two case reports of perineal hernia after laparoscopic abdominoperineal resection with a proposed modification to the operative technique. Ann R Coll Surg Engl 96: e9-e10. 